\title{
Uniform Stability of a Finite Difference Scheme for Transport Equations in Diffusive Regimes*
}

\author{
A. $\operatorname{Klar}^{\dagger} \quad$ A. Unterreiter
}

August 16, 2000

\begin{abstract}
An asymptotic preserving numerical scheme (with respect to diffusion scalings) for a linear transport equation is investigated. The scheme is adopted from a class of schemes developped in [6,7]. Stability is proven uniformly in the mean free path under a CFL type condition turning into a parabolic CFL condition in the diffusion limit.
\end{abstract}

\section{Introduction}

Transport equations and kinetic equations are used for a variety of applications, for example, to simulate radiative heat transfer processes or rarefied gas flows. Near to the continuum regimes the equations are approximated by macroscopic equations like diffusion equations or fluid dynamic equations. In recent years asymptotic preserving schemes for kinetic equations and transport equations have gained considerable attention in the literature. These schemes are used to treat singularly perturbed transport equations in situations with small mean free paths, i.e. in the above mentioned macroscopic limits. The general aim is to develop schemes allowing an underresolution in these limit situations. Schemes for instationary transport equations in the diffusion limit can be found, for example, in $[5],[6],[7],[11]$, see also the references therein. Schemes for different other transport equations with different macroscopic limits have been developped in $[4],[1],[8],[10],[9]$.

*This work has been supported by the TMR-network 'Asymptotic Methods in Kinetic Theory' and by Deutsche Forschungsgemeinschaft (DFG), grant KL 1105/7-1

${ }^{\dagger}$ Fachbereich Mathematik, Technische Universität Darmstadt, 64289 Darmstadt, Germany, (klar@mathematik.tu-darmstadt.de).

$\ddagger$ Fachbereich Mathematik, Universität Kaiserslautern, 67663 Kaiserslautern, Germany, (unterreiter@mathematik.uni-kl.de). 
Concerning the numerical analysis of these schemes proofs of uniform consistency with respect to small mean free path $\epsilon$ can be found in [6], [1], [7]. Furthermore, using homogenization theory for transport equations, a proof of uniform convergence (as $\epsilon \rightarrow 0$ ) for equations discretized spatially and in velocity is given in $[3,2]$.

In the present paper a numerical scheme for transport equations as developed in $[6,7]$ is considered. Numerical investigations of this scheme and proofs of uniform consistency can be found in $[6,7]$. The aim of the paper is to prove a uniform stability result for the method. The linear transport equation is introduced togehter with a time and space discretization. Linear stability is proven uniformly in $\epsilon$ using a careful direct analysis of the iterative scheme. The problem is tackled by a von Neumann analysis of the discrete system. This gives explicit and accurate estimates.

Under a $\epsilon$-dependent CFL type restriction the iterations are proven to be uniformly bounded. As $\epsilon$ tends to 0 the CFL type condition turns into a parabolic CFL condition as expected for the discretization of the limiting diffusion equation. For large mean free path, the CFL condition is the one adapted to the transport equation.

The paper is organized as follows. In Section 2 equations and schemes are introduced. Section 3 contains some definitions and the statement of the main result. In section 4 several preliminary results are established and, finally, section 5 contains the proof of the main result.

\section{Equations and Numerical Scheme}

Our model problem is the one dimensional linear transport equation with isotropic scattering,

$$
\epsilon^{2} \partial_{t} F+\epsilon v \partial_{x} F=\frac{1}{2} \int_{-1}^{1} F d v-F
$$

with density $F=F(x, v, t), x \in \mathbb{R}, v \in[-1,1]$ and $t \in[0, \infty)$.

We pass to the even-odd parity formulation by introducing for $v>0$ the even and odd functions

$$
\begin{aligned}
& f(v)=\frac{1}{2}(F(v)+F(-v)), \\
& g(v)=\frac{1}{2 \epsilon}(F(v)-F(-v)),
\end{aligned}
$$

i.e.

$$
\begin{aligned}
& F(v)=f(v)+\epsilon g(v), v>0 \\
& F(v)=f(-v)-\epsilon g(-v), v<0,
\end{aligned}
$$


such that (1) becomes for $v>0$,

$$
\begin{aligned}
\partial_{t} f+v \partial_{x} g & =\frac{1}{\epsilon^{2}}([f]-f), \quad[f]:=\int_{0}^{1} f d v \\
\partial_{t} g+\frac{v}{\epsilon^{2}} \partial_{x} f & =-\frac{1}{\epsilon^{2}} g .
\end{aligned}
$$

Remark 1. Concerning the limit $\epsilon \rightarrow 0$ in (2), (3) we obtain from a formal asymptotic expansion,

$$
f=\rho=[f], \quad g=-v \partial_{x} f
$$

where $\rho=\rho(x, t)$ fulfills the diffusion equation

$$
\partial_{t} \rho=\frac{1}{3} \partial_{x x} \rho
$$

We describe a scheme which is taken from a general class of schemes developed in [7], [5]. For the time discretization we use the time step $\Delta t \in \mathbb{R}^{+}$. The spatial step size is Deltatx. The time iterations approximating $f(x, v, n \Delta t)$ and $g(x, v, n \Delta t)$ are denoted by $f^{n}(x, v), g^{n}(x, v)$ for $n \in \mathbb{N}$ or $n=0$. Given $f^{n}, g^{n}$ we calculate $f^{n+1}, g^{n+1}$ as follows.

Algorithm. The discretization in time is obtained using a fractional step scheme. The spatial discretization is a simple first order discretization. For more complicated approaches, see for example [5].

Step 1: Approximate the solution of the system

$$
\begin{aligned}
\partial_{t} f+v \partial_{x} g & =0 \\
\partial_{t} g & =0
\end{aligned}
$$

by an explicit discretization, i.e. determine $f^{n+\frac{1}{2}}, g^{n+\frac{1}{2}}$ via

$$
\begin{aligned}
& f^{n+\frac{1}{2}}=f^{n}-\Delta t v D_{+} g^{n} \\
& g^{n+\frac{1}{2}}=g^{n}
\end{aligned}
$$

where $D_{+}$denotes the forward difference with step size $\Delta x$.

Step 2: Approximate the solution of the system

$$
\begin{aligned}
\partial_{t} f & =\frac{1}{\epsilon^{2}}([f]-f) \\
\partial_{t} g & =\frac{1}{\epsilon^{2}}\left(-v \partial_{x} f-g\right)
\end{aligned}
$$


by a semi-implicit discretization to treat the stiffness of the equations correctly, i.e. determine $f^{n+1}, g^{n+1}$ from $f^{n+\frac{1}{2}}, g^{n+\frac{1}{2}}$ via

$$
\begin{aligned}
& f^{n+1}=f^{n+\frac{1}{2}}+\frac{\Delta t}{\epsilon^{2}}\left(\left[f^{n+\frac{1}{2}}\right]-f^{n+1}\right) \\
& g^{n+1}=g^{n+\frac{1}{2}}+\frac{\Delta t}{\epsilon^{2}}\left[-v D_{-} f^{n+1}-g^{n+1}\right],
\end{aligned}
$$

where $D_{-}$denotes the backward difference with step size $\Delta x$.

Remark 2. We do not consider the velocity discretization. Usually a Gaussian quadrature is used.

We rewrite the recursion formula of Step 2 as

$$
\begin{aligned}
f^{n+1} & =A f^{n+\frac{1}{2}}+B\left[f^{n+\frac{1}{2}}\right] \\
g^{n+1} & =A g^{n+\frac{1}{2}}-B v D_{-} f^{n+1},
\end{aligned}
$$

with

$$
\begin{aligned}
A & :=\left(1+\frac{\Delta t}{\epsilon^{2}}\right)^{-1} \\
B & :=\frac{\Delta t}{\epsilon^{2}} A=1-A=\left(\frac{\epsilon^{2}}{\Delta t}+1\right)^{-1} .
\end{aligned}
$$

For the numerical analysis it is convenient to combine both steps in a single step,

$$
\begin{aligned}
& f^{n+1}=A\left(f^{n}-\Delta t v D_{+} g^{n}\right)+B\left[f^{n}-\Delta t v D_{+} g^{n}\right] \\
& g^{n+1}=A g^{n}-v A B D_{-} f^{n+1}-v B^{2} D_{-}\left[f^{n+1}\right]
\end{aligned}
$$

or

$$
g^{n+1}=A g^{n}-v A B D_{-}\left(f^{n}-\Delta t v D_{+} g^{n}\right)-v B^{2} D_{-}\left[f^{n}-\Delta t v D_{+} g^{n}\right] .
$$

Scheme (7) will be investigated in the following. Uniform consistency of similiar schemes has been considered in [7] and [6]. Here we will prove a uniform (in $\epsilon$ ) stability result.

Remark 3. Keeping $\Delta t$ fixed and considering the limit $\epsilon \rightarrow 0$ of (7) we have $A \rightarrow 0, B \rightarrow 1$ as $\epsilon \rightarrow 0$ and we obtain the scheme

$$
\begin{aligned}
f^{n+1} & =\left[f^{n+1}\right]=\left[f^{n}-\Delta t v D_{+} g^{n}\right] \\
g^{n+1} & =-v D_{-}\left[f^{n+1}\right],
\end{aligned}
$$

i.e. in terms of $\rho^{n}=\left[f^{n}\right]$,

$$
\rho^{n+1}=\rho^{n}+\frac{1}{3} \Delta t D_{+} D_{-} \rho^{n}
$$

which is a straightforward explicit discretization of the diffusion equation (4). 


\section{The main result}

In this section we state a theorem on uniform stability for (7). The proof is settled on a von Neumann stability analysis.

The recursion scheme (7) involves two positive discretization parameters $\Delta t, \Delta x$ (which enters via $D_{ \pm}$) and the scaled mean free path $\epsilon \in(0, \infty)$. In the sequel it is assumed that $\Delta t, \Delta x, \epsilon$ satisfy the following condition.

Definition 1. $\Delta t, \Delta x$ and $\epsilon$ fulfill the "transport $C F L$ condition" iff

$$
\frac{\Delta t}{(\Delta x)^{2}} \frac{\Delta t}{\epsilon^{2}+\Delta t}<\frac{1}{2}
$$

Remark 4. Condition (8) is equivalent to

$$
\frac{\Delta t}{(\Delta x)^{2}}<\frac{\epsilon^{2}+\Delta t}{2 \Delta t}
$$

or

$$
\frac{\Delta t}{\Delta x}<\sqrt{\frac{\epsilon^{2}+\Delta t}{2}}
$$

For $\epsilon^{2} \ll \Delta t$ condition (9) reduces to a "parabolic CFL condition",

$$
\frac{\Delta t}{(\Delta x)^{2}}<\frac{1}{2}
$$

and in case $\epsilon^{2} \gg \Delta t$, condition (10) reduces to

$$
\frac{\Delta t}{\Delta x}<\frac{\epsilon}{\sqrt{2}}
$$

which is for fixed $\epsilon$ a "hyperbolic CFL condition".

Remark 5. Introducing

$$
\rho:=\frac{\Delta t}{\epsilon^{2}}, \quad \text { i.e. } \Delta t=\rho \epsilon^{2},
$$

the transport CFL condition (8) holds if and only if

$$
\exists \delta \in \mathbb{R}^{+}: \quad(\Delta x)^{2}=2 \rho^{2} \frac{1+\delta}{1+\rho} \cdot \epsilon^{2} .
$$

Here, $\rho \ll 1$ corresponds to the fine resolved case $\Delta t \ll \epsilon^{2}$ and $1 \ll \rho$ corresponds to the under-resolved case $\epsilon^{2} \ll \Delta t$. 
Now we shall give the recursion (7) a well-defined meaning by introducing sets of functions on which the recursion operator of (7) acts.

We put

$$
\begin{gathered}
M:=\{\phi: \mathbb{R} \times(0,1) \rightarrow \mathbb{C}: \phi \text { is measurable }\} \\
M^{0,2}:=\left\{\phi \in M:\left(\forall v \in(0,1): \int_{\mathbb{R}}|\phi(x, v)|^{2} d x<\infty\right)\right\} .
\end{gathered}
$$

We are heading towards a von Neumann stability analysis of (7). This requires for a notation for the Fourier transform of $\phi \in M^{0,2}$ with respect to $x$. We put

$$
\hat{\phi}(\xi, v):=\frac{1}{\sqrt{2 \pi}} \int_{\mathbb{R}} \phi(x, v) \exp (-i x \xi) d x, \quad(\xi, v) \in \mathbb{R} \times(0,1) .
$$

For later reference we introduce the space

$$
M^{1,2}:=\left\{\phi \in M^{0,2}:\left(\forall v \in(0,1): \int_{\mathbb{R}}\left(1+|\xi|^{2}\right)|\hat{\phi}(\xi, v)|^{2} d \xi<\infty\right)\right\} .
$$

Remark 6. Due to the standard theory of Sobolev spaces we have $\phi(., v) \in H^{1}(\mathbb{R})$ for all $\phi \in M^{1,2}$ and for all $v \in(0,1)$ and

$$
\|\phi(., v)\|_{H^{1}(\mathbb{R})}^{2} \leq K \int_{\mathbb{R}}\left(1+|\xi|^{2}\right)|\hat{\phi}(\xi, v)|^{2} d \xi
$$

where $K$ is independent of $\phi \in M^{1,2}$.

Applying the Fourier transform (with respect to $x$ ) on (7) we obtain the recursion scheme

$$
\begin{aligned}
& \hat{f}^{n+1}=A\left(\hat{f}^{n}+v \alpha \hat{g}^{n}\right)+B\left[\hat{f}^{n}+\alpha v \hat{g}^{n}\right], \\
& \hat{g}^{n+1}=A\left(\hat{g}^{n}+v \beta \hat{f}^{n}-\Theta v^{2} \hat{g}^{n}\right)+B v \beta\left[\hat{f}^{n}+\alpha v \hat{g}^{n}\right],
\end{aligned}
$$

where

$$
\begin{gathered}
\alpha=\alpha(\xi, \Delta t, \Delta x)=\frac{\Delta t}{\Delta x}\left(1-\mathrm{e}^{i \xi \Delta x}\right)=\xi \Delta t H(\xi \Delta x) \\
\beta=\beta(\xi, \Delta t, \Delta x, \epsilon)=\frac{B}{\Delta x}\left(\mathrm{e}^{-i \xi \Delta x}-1\right)=-\frac{\xi \Delta t}{\epsilon^{2}+\Delta t} \bar{H}(\xi \Delta x), \\
\Theta=\Theta(\xi, \Delta t, \Delta x, \epsilon)=-\alpha \beta=\frac{\Delta t}{(\Delta x)^{2}} \frac{\Delta t}{\epsilon^{2}+\Delta t}(2-2 \cos (\xi \Delta x)),
\end{gathered}
$$


and the holomorphic function $H$ is given by

$$
H: \mathbb{C} \rightarrow \mathbb{C}, \quad H(z)=\left\{\begin{array}{cc}
\frac{1-\exp (i z)}{z} & , z \neq 0 \\
-i & , z=0
\end{array},\right.
$$

is bounded on $\mathbb{R}$ with $|H(\sigma)|<1$ for all $\sigma \in \mathbb{R}$ with $\sigma \neq 0$, and $|H(0)|=1$.

Remark 7. For all positive $\Delta t, \Delta x, \epsilon$ we have

$$
0=\inf _{\xi \in \mathbb{R}} \Theta(\xi, \Delta t, \Delta x, \epsilon)<\sup _{\xi \in \mathbb{R}} \Theta(\xi, \Delta t, \Delta x, \epsilon)=\frac{4 \Delta t}{(\Delta x)^{2}} \frac{\Delta t}{\epsilon^{2}+\Delta t},
$$

which highlights the distinctive importance of the value of $\Theta$ : The transport $C F L$ condition is equivalent to

$$
\sup _{\xi \in \mathbb{R}} \Theta(\xi, \Delta t, \Delta x, \epsilon)<2 .
$$

It is convenient to introduce for $f, g \in M$ and $\hat{f}, \hat{g} \in M$ the notations

$$
\mathfrak{f}=(f, g), \quad \hat{\mathfrak{f}}=(\hat{f}, \hat{g}) .
$$

We rewrite recursion (13) as

$$
\hat{\mathfrak{f}}^{n+1}=\left(A T+B T_{0}\right) \hat{\mathfrak{f}}^{n}
$$

with $A, B \in(0,1), A+B=1$ as above and $n \in \mathbb{N}$. The linear operators $T$ and $T_{0}$ depend on the parameters $\alpha, \beta$. We have for $\hat{\mathfrak{f}}=(\hat{f}, \hat{g}) \in M \times M$ and for $(\xi, v) \in \mathbb{R} \times(0,1)$,

$$
T(\hat{\mathfrak{f}})(\xi, v)=\left(\hat{f}(\xi, v)+\alpha v \hat{g}(\xi, v), \beta v \hat{f}(\xi, v)+\left(1-\Theta v^{2}\right) \hat{g}(\xi, v)\right),
$$

and

$$
T_{0}(\hat{\mathfrak{f}})(\xi, v)=([\hat{f}+\alpha v \hat{g}](\xi), \beta v[\hat{f}+\alpha v \hat{g}](\xi)) .
$$

Remark 8. We obviously have $T, T_{0}: M \rightarrow M$ and - due to the linear dependence of $\alpha$ on $\xi$ - we have: $T(\hat{\mathfrak{f}}), T_{0}(\hat{\mathfrak{f}}) \in M^{0,2}$ for all $\mathfrak{f} \in M^{1,2}$. Later on we will actually show $T^{n}(\hat{\mathfrak{f}}), T_{0}^{n}(\hat{\mathfrak{f}}) \in M^{0,2}$ for all $\mathfrak{f} \in M^{1,2}$ and for all $n \in \mathbb{N}$.

Our aim is to prove uniform boundedness in suitable norms of the iterations $\mathfrak{f}^{n}=\left(f^{n}, g^{n}\right)$ for all $n \in \mathbb{N}$ and $\epsilon \geq 0$.

The results will depend on point-wise estimates of $\hat{\mathfrak{f}}^{n}$. Let us highlight the argumentation at hand of the formal limiting problem when $\epsilon$ is set to zero. In this situation recursion (17) reduces to $\hat{\mathfrak{f}}^{n+1}=\left(\hat{f}^{n+1}, \hat{g}^{n+1}\right)=T_{0} \hat{\mathfrak{f}}^{n}$, this means

$$
\begin{aligned}
& \hat{f}^{n+1}=\left[\hat{f}^{n}+\alpha v \hat{g}^{n}\right] \\
& \hat{g}^{n+1}=v \beta\left[\hat{f}^{n}+\alpha v \hat{g}^{n}\right]=v \beta\left[\hat{f}^{n+1}\right]
\end{aligned}
$$


with

$$
\beta=\beta(\xi, \Delta t, \Delta x, \epsilon=0)=-\xi \bar{H}(\xi \Delta x) .
$$

This yields

$$
\hat{f}^{n+1}=\left(1-\Theta\left[v^{2}\right]\right)\left[\hat{f}^{n}\right]=\left(1-\frac{\Theta}{3}\right)\left[\hat{f}^{n}\right],
$$

where

$$
\Theta=\Theta(\xi, \Delta t, \Delta x, \epsilon=0)=\frac{\Delta t}{(\Delta x)^{2}}(1-\cos (\xi \Delta x)) .
$$

Thus, we have the point-wise estimates

$$
\begin{aligned}
\left|\hat{f}^{n}\right|(\xi, v) & =\left|1-\frac{\Theta}{3}\right|^{n}\left|\left[\hat{f}^{0}\right]\right|(\xi) \\
\left|\hat{g}^{n}\right|(\xi, v) & \leq|v||\xi|\left|1-\frac{\Theta}{3}\right|^{n}\left|\left[\hat{f}^{0}\right]\right|(\xi)
\end{aligned}
$$

in particular, whenever

$$
\sup _{\xi \in \mathbb{R}} \Theta(\xi, \Delta t, \Delta x, \epsilon=0) \leq 6,
$$

which is the case if and only if the usual parabolic CFL condition for the diffusion equation (4)

$$
\frac{\Delta t}{(\Delta x)^{2}} \leq \frac{3}{2}
$$

holds, then for all $n \in \mathbb{N}$,

$$
\begin{aligned}
\left|\hat{f}^{n}\right|(\xi, v) & \leq\left|\left[\hat{f}^{0}\right]\right|(\xi) \\
\left|\hat{g}^{n}\right|(\xi, v) & \leq|\xi|\left|\left[\hat{f}^{0}\right]\right|(\xi) .
\end{aligned}
$$

From (21), (22) we obtain under the assumption that all involved integrals are finite for all $n \in \mathbb{N}$ and for all $v \in(0,1)$ the estimates

$$
\begin{aligned}
\int_{\mathbb{R}}\left|\hat{f}^{n}\right|^{2}(\xi, v) d \xi & \leq \int_{\mathbb{R} \times(0,1)}\left|\hat{f}^{0}\right|^{2}(\xi, u) d(\xi, u) \\
\int_{\mathbb{R}}\left|\hat{g}^{n}\right|^{2}(\xi, v) d \xi & \leq \int_{\mathbb{R} \times(0,1)}|\xi|^{2}\left|\hat{f}^{0}\right|^{2}(\xi, u) d(\xi, u),
\end{aligned}
$$

thus for all $n \in \mathbb{N}$ and for all $v \in(0,1)$,

$$
\int_{\mathbb{R}}\left(\left|\hat{f}^{n}\right|^{2}+\left|\hat{g}^{n}\right|^{2}\right)(\xi, v) d \xi \leq \int_{\mathbb{R} \times(0,1)}\left(1+|\xi|^{2}\right)\left|\hat{f}^{0}\right|^{2}(\xi, u) d(\xi, u) .
$$


This motivates the introduction of the anisotropic semi-norms

$$
\begin{aligned}
& \|.\|_{1 ; 1}: M^{1,2} \times M^{1,2} \rightarrow \mathbb{R}_{0}^{+} \cup\{\infty\}, \\
& \|(f, g)\|_{1 ; 1}:=\sqrt{\int_{\mathbb{R} \times(0,1)}\left(1+|\xi|^{2}\right)\left(|\hat{f}|^{2}+|\hat{g}|^{2}\right)(\xi, u) d(\xi, u)}, \\
& \|.\|_{0 ; \infty}: M^{0,2} \times M^{0,2} \rightarrow \mathbb{R}_{0}^{+} \cup\{\infty\}, \\
& \|(f, g)\|_{0 ; \infty}:=\sup _{v \in(0,1)} \sqrt{\int_{\mathbb{R}}\left(|f|^{2}+|g|^{2}\right)(\xi, v) d \xi},
\end{aligned}
$$

and to define the anisotropic spaces of Sobolev type,

$$
\begin{gathered}
W^{1 ; 1}:=\mathcal{L}^{2}\left((0,1): \mathcal{H}^{1}(\mathbb{R}) \times \mathcal{H}^{1}(\mathbb{R})\right)=\left\{(f, g) \in M^{1,2} \times M^{1,2}:\|(f, g)\|_{1 ; 1}<\infty\right\}, \\
W^{0 ; \infty}:=\mathcal{L}^{\infty}\left((0,1): \mathcal{L}^{2}(\mathbb{R}) \times \mathcal{L}^{2}(\mathbb{R})\right)=\left\{(f, g) \in M^{0,2} \times M^{0,2}:\|(f, g)\|_{0 ; \infty}<\infty\right\} .
\end{gathered}
$$

Assuming the validity of (20) (here no further condition on the parameters $\Delta t, \Delta x, \epsilon$ are required) we can re-write (25) in the more convenient form

$$
\left\|\mathfrak{f}^{n}\right\|_{0 ; \infty} \leq\left\|\mathfrak{f}^{0}\right\|_{1 ; 1}, \quad n \in \mathbb{N}
$$

where we made use of the fact that the Fourier transform maps $L^{2}(\mathbb{R})$ isometrically into itself.

For the general case we introduce additionally

$$
\begin{aligned}
W^{2}:=\mathcal{L}^{2}(\mathbb{R} \times & (0,1)) \times \mathcal{L}^{2}(\mathbb{R} \times(0,1)) \\
& =\left\{(f, g) \in M \times M: \int_{\mathbb{R} \times(0,1)}\left(|f|^{2}+|g|^{2}\right)(\xi, v) d(\xi, v)<\infty\right\}
\end{aligned}
$$

equipped with the canonical semi-norm

$$
\|(f, g)\|_{2}:=\sqrt{\int_{\mathbb{R} \times(0,1)}\left(|f|^{2}+|g|^{2}\right)(\xi, v) d(\xi, v)} .
$$

Our main result is the following theorem:

Theorem 1. Let $\Delta x, \Delta t, \epsilon \in \mathbb{R}^{+}$. Let $\mathfrak{f}^{0} \in W^{1 ; 1}$ and let the sequence $\left(\mathfrak{f}^{n}\right)_{n \in \mathbb{N}}=$ $\left(\left(f^{n}, g^{n}\right)\right)_{n \in \mathbb{N}}$ be defined by (7) (with initial value $\left.\mathfrak{f}^{0}\right)$.

Assume $\Delta t, \Delta x, \epsilon$ satisfy (8). Then: 
a) $\mathfrak{f}^{n} \in W^{2}$ for all $n \in \mathbb{N}$ and

$$
\left\|\mathfrak{f}^{n}\right\|_{2} \leq 2\left(42 \sqrt{\epsilon^{2}+\Delta t}+15+\frac{\sqrt{2} \epsilon^{2}}{\left(\epsilon^{2}+\Delta t\right)^{3 / 2}}\right)\left\|\mathfrak{f}^{0}\right\|_{1 ; 1} .
$$

b) If $\mathfrak{f}^{0} \in W^{1 ; 1} \cap W^{0 ; \infty}$, then $\mathfrak{f}^{n} \in W^{0 ; \infty}$ for all $n \in \mathbb{N}$ and

$$
\begin{aligned}
&\left\|\mathfrak{f}^{n}\right\|_{0 ; \infty} \leq \sqrt{2}\left(2+2 \sqrt{\epsilon^{2}+\Delta t}+\frac{\sqrt{2} \epsilon^{2}}{\left(\epsilon^{2}+\Delta t\right)^{3 / 2}}\right)\left\|\mathfrak{f}^{0}\right\|_{0 ; \infty} \\
&+2\left(15+24 \sqrt{\epsilon^{2}+\Delta t}\right)\left\|\mathfrak{f}^{0}\right\|_{1 ; 1} .
\end{aligned}
$$

Theorem 1 allows for the derivation of several stability results for (7) independently of $\epsilon$. As examples, we deduce

Corollary 1. Let $M, \epsilon_{0}$ be positive constants. Then there is a positive constant $C_{0}=C_{0}\left(M, \epsilon_{0}\right)$ such that for all $\Delta x, \Delta t, \epsilon \in \mathbb{R}^{+}$:

If $\Delta t, \Delta x, \epsilon$ satisfy (8), if $\Delta t+\epsilon \leq M$ and if $\epsilon \leq \epsilon_{0} \Delta t$, then the following estimates hold for any sequence $\left(\mathfrak{f}^{n}\right)_{n \in \mathbb{N}}=\left(\left(f^{n}, g^{n}\right)\right)_{n \in \mathbb{N}}$ defined by (7) with initial value $\mathfrak{f}^{0} \in W^{1 ; 1}$ :

a) $\mathfrak{f}^{n} \in W^{2}$ for all $n \in \mathbb{N}$ and $\left\|\mathfrak{f}^{n}\right\|_{2} \leq C_{0}\left\|\mathfrak{f}^{0}\right\|_{1 ; 1}$.

b) If $\mathfrak{f}^{0} \in W^{1 ; 1} \cap W^{0 ; \infty}$, then $\mathfrak{f}^{n} \in W^{0 ; \infty}$ for all $n \in \mathbb{N}$ and

$$
\left\|\mathfrak{f}^{n}\right\|_{0 ; \infty} \leq C_{0}\left(\left\|\mathfrak{f}^{0}\right\|_{0 ; \infty}+\left\|\mathfrak{f}^{0}\right\|_{1 ; 1}\right) .
$$

Corollary 2. Let $M, \epsilon_{1}$ be positive constants. Then there is a positive constant $C_{1}=C_{1}\left(M, \epsilon_{1}\right)$ such that for all $\Delta x, \Delta t, \epsilon \in \mathbb{R}^{+}$:

If $\Delta t, \Delta x, \epsilon$ satisfy (8), if $\Delta t+\epsilon \leq M$ and if $\epsilon_{1} \leq \epsilon$, then the following estimates hold for any sequence $\left(\mathfrak{f}^{n}\right)_{n \in \mathbb{N}}=\left(\left(f^{n}, g^{n}\right)\right)_{n \in \mathbb{N}}$ defined by (7) with initial value $\mathfrak{f}^{0} \in W^{1 ; 1}$ :

a) $\mathfrak{f}^{n} \in W^{2}$ for all $n \in \mathbb{N}$ and $\left\|\mathfrak{f}^{n}\right\|_{2} \leq C_{1}\left\|\mathfrak{f}^{0}\right\|_{1 ; 1}$.

b) If $\mathfrak{f}^{0} \in W^{1 ; 1} \cap W^{0 ; \infty}$, then $\mathfrak{f}^{n} \in W^{0 ; \infty}$ for all $n \in \mathbb{N}$ and

$$
\left\|\mathfrak{f}^{n}\right\|_{0 ; \infty} \leq C_{1}\left(\left\|\mathfrak{f}^{0}\right\|_{0 ; \infty}+\left\|\mathfrak{f}^{0}\right\|_{1 ; 1}\right) \text {. }
$$

Remark 9. It is remarkable that although the scheme is developed based on consideration of the diffusive limit $\epsilon$ tending to 0, the transport CFL condition (8) is sufficient to guarantee stability also for large $\epsilon$. In particular, for large mean free paths the time step is not any more restricted by a parabolic CFL condition related to the limiting diffusion equation, but by the hyperbolic CFL condition (12) related to the transport equation. 
Remark 10. The transport CFL condition (8) is seemingly not optimal. For example for $\epsilon$ tending to 0 we have

$$
\frac{\Delta t}{(\Delta x)^{2}}<\frac{1}{2}
$$

However, as the direct analysis for $\epsilon=0$ shows, actually, the correct restriction is the parabolic CFL (20)

$$
\frac{\Delta t}{(\Delta x)^{2}}<\frac{3}{2}
$$

Remark 11. The conditions $\epsilon_{0} \leq \epsilon \leq M$ or $\epsilon \leq \epsilon_{1} \Delta t$, cover the fine resolved and under-resolved cases.

\section{Preliminaries}

The main ingredients of the proof of Theorem 1 are investigations of recursion formulae. These investigations require several preliminary estimates.

Lemma 2. Let $\sigma \in(0,2)$. We put

$$
\psi:(0,1) \rightarrow(0,1), \quad \psi(v)=\arccos \left(1-\frac{\sigma v^{2}}{2}\right)
$$

Then for all $n \in \mathbb{N}$,

$$
\left|\int_{0}^{1}\left(\cos (n \psi(v))-\sin (n \psi(v)) \frac{1-\cos (\psi(v))}{\sin (\psi(v))}\right) d v\right| \leq 1 .
$$

Proof. Introducing $t:=\cos (\psi(v))$ as new variable we obtain

$$
\begin{gathered}
\int_{0}^{1}\left(\cos (n \psi(v))-\sin (n \psi(v)) \frac{1-\cos (\psi(v))}{\sin (\psi(v))}\right) d v \\
=\frac{1}{\sqrt{2 \sigma}} \int_{0}^{\arccos ((2-\sigma) / 2)}(\sqrt{1+\cos (t)} \cdot \cos (n t)-\sqrt{1-\cos (t)} \cdot \sin (n t)) d t \\
=\frac{1}{\sqrt{\sigma}} \int_{0}^{\arccos ((2-\sigma) / 2)}(\cos (t / 2) \cdot \cos (n t)-\sin (t / 2) \cdot \sin (n t)) d t \\
=\frac{1}{\sqrt{\sigma}} \frac{\sin ((n+(1 / 2) \arccos ((2-\sigma) / 2))}{n+(1 / 2)}=\frac{\sin ((n+(1 / 2)) \psi)}{(n+(1 / 2)) \sqrt{2(1-\cos (\psi))}} \\
=\frac{\sin ((n+(1 / 2)) \psi)}{(n+(1 / 2)) \psi} \cdot \frac{\psi / 2}{\sin (\psi / 2)} \in[-1,+1]
\end{gathered}
$$


Lemma 3. Let $\left(c_{n}\right)_{n \in \mathbb{N}}$ and $\left(\gamma_{n}\right)_{n \in \mathbb{N}}$ be complex sequences. Define a complex sequence $\left(\kappa_{n}\right)_{n \in \mathbb{N}}$ by recursion via

$$
\kappa_{0}=c \in \mathbb{C}, \quad \kappa_{n+1}=c_{n}+\left(\kappa_{0} \cdot \gamma_{n-1}+\kappa_{1} \cdot \gamma_{n-2}+\ldots+\kappa_{n-1} \cdot \gamma_{0}\right)
$$

Assume

$$
\sum_{k=0}^{\infty}\left|c_{k}\right|<\infty, \quad \sum_{k=0}^{\infty}\left|\gamma_{k}\right| \leq 1 .
$$

Then the sequence $\left(\kappa_{n}\right)_{n \in \mathbb{N}}$ is bounded, more precisely,

$$
\forall n \in \mathbb{N}: \quad\left|\kappa_{n}\right| \leq\left|\kappa_{0}\right|+\left(\left|c_{0}\right|+\ldots+\left|c_{n-1}\right|\right) .
$$

Proof. We prove (27) by induction. There is nothing to do in case $n=0$. To pass from $n$ to $n+1$, we calculate

$$
\begin{aligned}
\left|\kappa_{n+1}\right| & =\left|c_{n}+\sum_{j=0}^{n-1} \kappa_{j} \cdot \gamma_{n-1-j}\right| \leq\left|c_{n}\right|+\sum_{j=0}^{n-1}\left|\kappa_{j}\right| \cdot\left|\gamma_{n-1-j}\right| \\
& \leq\left|c_{n}\right|+\max \left\{\left|\kappa_{0}\right|, \ldots,\left|\kappa_{n-1}\right|\right\} \sum_{j=0}^{n-1}\left|\gamma_{j}\right| \leq\left|c_{n}\right|+\left|\kappa_{0}\right|+\left|c_{0}\right|+\ldots+\left|c_{n-1}\right| .
\end{aligned}
$$

Furthermore, we require the following result about the recursion scheme (7) when $A$ is set to 1 (or equivalently, when $B$ is set to 0 ).

Lemma 4. Let $\xi \in \mathbb{R}$ and let $\Delta t, \Delta x, \epsilon$ be positive real numbers. Let $\alpha, \beta, \Theta$ be as in (14), (15), (16), respectively. For $\left(\hat{f}_{0}, \hat{g}_{0}\right) \in M \times M$ let $T$ as in (18), i.e. for $(\xi, v) \in \mathbb{R} \times(0,1)$ in vector notation,

$$
T\left(\begin{array}{c}
\hat{f}_{0} \\
\hat{g}_{0}
\end{array}\right)(\xi, v)=\left(\begin{array}{cc}
1 & \alpha v \\
\beta v & 1-\Theta v^{2}
\end{array}\right)\left(\begin{array}{l}
\hat{f}_{0}(\xi, v) \\
\hat{g}_{0}(\xi, v)
\end{array}\right) .
$$

For $n \in \mathbb{N}$ let

$$
\left(\begin{array}{c}
\hat{f}_{n} \\
\hat{g}_{n}
\end{array}\right)=T^{n}\left(\begin{array}{c}
\hat{f}_{0} \\
\hat{g}_{0}
\end{array}\right) .
$$

Assume $\Delta t, \Delta x, \epsilon$ satisfy the transport $C F L$ condition (8).

Then for all $n \in \mathbb{N}$ and for all $(\xi, v) \in \mathbb{R} \times(0,1)$,
1. $\left|\hat{f}_{n}\right|(\xi, v) \leq\left(2\left|\hat{f}_{0}\right|+\sqrt{2\left(\epsilon^{2}+\Delta t\right)}\left|\hat{g}_{0}\right|\right)(\xi, v)$.
2. $\left|\hat{g_{n}}\right|(\xi, v) \leq\left(\frac{\sqrt{2}}{\sqrt{\epsilon^{2}+\Delta t}}\left|\hat{f}_{0}\right|+2\left|\hat{g_{0}}\right|\right)(\xi, v)$. 
3. $\left|\alpha v \hat{g_{n}}\right|(\xi, v) \leq\left(2\left|\hat{f}_{0}\right|+4 \sqrt{\epsilon^{2}+\Delta t}\left|\hat{g}_{0}\right|\right)(\xi, v)$.

4. If $\hat{f}_{0}=1$ and if $\hat{g}_{0}(\xi, v)=\beta v$, then

$$
\left|\hat{f}_{n}\right|(\xi, v) \leq 2,\left|\hat{g_{n}}\right|(\xi, v) \leq 3|\xi|, \quad\left|\int_{0}^{1}\left(\hat{f}_{n}(\xi, s)+\alpha s \hat{g_{n}}(\xi, s)\right) d s\right| \leq 1 .
$$

Proof. We recall: If $\Delta t, \Delta x, \epsilon$ satisfy (8), then $\sup _{\xi \in \mathbb{R}} \Theta(\xi, \Delta t, \Delta x, \epsilon)<2$. We shall use this estimate frequently.

We keep $(\xi, v) \in \mathbb{R} \times(0,1)$ fixed and introduce the $2 \times 2$ matrix

$$
R:=R(\alpha, \beta, \Theta, v):=\left(\begin{array}{cc}
1 & \alpha v \\
\beta v & 1-\Theta v^{2}
\end{array}\right) .
$$

Then we have for all $n \in \mathbb{N}$ or $n=0$,

$$
T^{n}\left(\begin{array}{c}
\hat{f}_{0} \\
\hat{g}_{0}
\end{array}\right)(\xi, v)=R^{n} \cdot\left(\begin{array}{c}
\hat{f}_{0}(\xi, v) \\
\hat{g}_{0}(\xi, v)
\end{array}\right) .
$$

If $\Theta=0$, then $\xi=0$ and therefore $\alpha=\beta=0$ as well. In this case $R$ is the identity matrix and the proof of the lemma is straight-forward.

Let us assume $\Theta>0$ henceforth.

The eigenvalues of $R$ are

$$
\lambda_{1,2}(v)=\left(1-\frac{\Theta v^{2}}{2}\right) \pm \sqrt{\left(\frac{\Theta v^{2}}{2}\right)^{2}-\Theta v^{2}} .
$$

Since $a:=\Theta v^{2} / 2<1$, we have $2 a-a^{2}>0$ such that

$$
\lambda_{1,2}=(1-a) \pm i \sqrt{2 a-a^{2}},
$$

i.e. $R$ has two distinct, non-real, complex conjugate eigenvalues

$$
\lambda_{1}=\lambda:=(1-a)+i \sqrt{2 a-a^{2}}, \quad \lambda_{2}=\bar{\lambda} .
$$

Hence

$$
R^{n}=B\left(\begin{array}{cc}
\lambda^{n} & 0 \\
0 & \bar{\lambda}^{n}
\end{array}\right) B^{-1} .
$$

Since $|\lambda|=1$ we have $\lambda:=e^{i \theta}$ for some $\theta \in(0,2 \pi)$. Since $\cos (\theta)=1-a>0$ and $\sin (\theta)=\sqrt{2 a-a^{2}}>0$, we have $\theta \in(0, \pi / 2)$. Furthermore,

$$
v=\frac{2}{\sqrt{\Theta}} \sin (\theta / 2) \text {. }
$$


Hence for all $n \in \mathbb{N}$,

$$
\begin{aligned}
& R^{n}=\frac{i}{2 \alpha v \sin (\theta)}\left(\begin{array}{cc}
-\alpha v & -\alpha v \\
1-e^{i \theta} & 1-e^{-i \theta}
\end{array}\right)\left(\begin{array}{cc}
e^{n i \theta} & 0 \\
0 & e^{-n i \theta}
\end{array}\right)\left(\begin{array}{cc}
1-e^{-i \theta} & \alpha v \\
-1+e^{i \theta} & -\alpha v
\end{array}\right) \\
& =\frac{i}{2 \alpha v \sin (\theta)}\left(\begin{array}{cc}
-\alpha v & -\alpha v \\
1-e^{i \theta} & 1-e^{-i \theta}
\end{array}\right)\left(\begin{array}{cc}
e^{n i \theta}-e^{(n-1) i \theta} & \alpha v e^{n i \theta} \\
-e^{-n i \theta}+e^{-(n-1) i \theta} & -\alpha v e^{-n i \theta}
\end{array}\right) \\
& =\left(\begin{array}{cc}
\frac{\sin (n \theta)-\sin ((n-1) \theta)}{\sin (\theta)} & \alpha v \frac{\sin (n \theta)}{\sin (\theta)} \\
-\frac{2 \sin (n \theta)-\sin ((n-1) \theta)-\sin ((n+1) \theta)}{\alpha v \sin (\theta)} & -\frac{\sin (n \theta)-\sin ((n+1) \theta)}{\sin (\theta)}
\end{array}\right) \\
& =\left(\begin{array}{cc}
\frac{\sin (n \theta)-\sin ((n-1) \theta)}{\sin (\theta)} & \frac{2 \alpha}{\sqrt{\Theta}} \frac{\sin (\theta / 2) \sin (n \theta)}{\sin (\theta)} \\
-\frac{\sqrt{\Theta}}{2 \alpha} \frac{2 \sin (n \theta)-\sin ((n-1) \theta)-\sin ((n+1) \theta)}{\sin (\theta / 2) \sin (\theta)} & \frac{\sin (n \theta)-\sin ((n+1) \theta)}{\sin (\theta)}
\end{array}\right) \\
& =\left(\begin{array}{cc}
\cos (n \theta)+\sin (n \theta) \frac{1-\cos (\theta)}{\sin (\theta)} & \frac{2 \alpha}{\sqrt{\Theta}} \sin (n \theta) \frac{\sin (\theta / 2)}{\sin (\theta)} \\
-\frac{\sqrt{\Theta}}{\alpha} \sin (n \theta) \frac{1-\cos (\theta)}{\sin (\theta / 2) \sin (\theta)} & \cos (n \theta)-\sin (n \theta) \frac{1-\cos (\theta)}{\sin (\theta)}
\end{array}\right) \\
& =:\left(\begin{array}{cc}
R_{n ; 11} & R_{n ; 12} \\
R_{n ; 21} & R_{n ; 22}
\end{array}\right) .
\end{aligned}
$$

Since $\theta=\theta \in(0, \pi / 2)$ we have

$$
\left|R_{n ; 11}\right|,\left|R_{n ; 22}\right| \leq 2 .
$$

Furthermore, we have for all $n \in \mathbb{N}$,

$$
\begin{gathered}
R_{n ; 12}=\frac{2 \alpha}{\sqrt{\Theta}} \sin (n \theta) \frac{\sin (\theta / 2)}{\sin (\theta)}=\alpha v \frac{\sin (n \theta)}{\sin (\theta)}=\alpha v \frac{\sin (n \theta)}{\sqrt{\Theta} v \sqrt{1-\frac{\Theta v^{2}}{4}}} \\
=\frac{\alpha}{\sqrt{\Theta}} \frac{\sin (n \theta)}{\sqrt{1-\frac{\Theta v^{2}}{4}}}=\frac{(\xi \Delta t H(\xi \Delta x))\left(\Delta x \sqrt{\epsilon^{2}+\Delta t}\right)}{\Delta t \sqrt{2(1-\cos (\xi \Delta x))} \frac{\sin (n \theta)}{\sqrt{1-\frac{\Theta v^{2}}{4}}}} \\
=\sqrt{\epsilon^{2}+\Delta t} \frac{\xi \Delta x H(\xi \Delta x)}{\sqrt{2(1-\cos (\xi \Delta x))}} \frac{\sin (n \theta)}{\sqrt{1-\frac{\Theta v^{2}}{4}}} \\
=\sqrt{\epsilon^{2}+\Delta t} \frac{H(\xi \Delta x)}{|H(\xi \Delta x)|} \frac{\sin (n \theta)}{\sqrt{1-\frac{\Theta v^{2}}{4}}}
\end{gathered}
$$

and in analogy,

$$
R_{n ; 21}=-\frac{1}{\sqrt{\epsilon^{2}+\Delta t}} \frac{|H(\xi \Delta x)|}{H(\xi \Delta x)} \frac{\sin (n \theta)}{\sqrt{1-\frac{\Theta v^{2}}{4}}}
$$


hence

$$
\begin{aligned}
& \left|R_{n ; 12}\right| \leq \sqrt{2\left(\epsilon^{2}+\Delta t\right)} \\
& \left|R_{n ; 21}\right| \leq \frac{\sqrt{2}}{\sqrt{\epsilon^{2}+\Delta t}} .
\end{aligned}
$$

Since for all $n \in \mathbb{N}$,

$$
\begin{aligned}
& \hat{f_{n}}(\xi, v)=R_{n ; 11} \hat{f}_{0}(\xi, v)+R_{n ; 12} \hat{g}_{0}(\xi, v) \\
& \hat{g_{n}}(\xi, v)=R_{n ; 21} \hat{f}_{0}(\xi, v)+R_{n ; 22} \hat{g}_{0}(\xi, v)
\end{aligned}
$$

statements 1. and 2. of the lemma follow from (29), (32), (33).

Now we calculate for all $n \in \mathbb{N}$,

$$
\alpha v R_{n ; 21}=-v \sqrt{\Theta} \sin (n \theta) \frac{1-\cos (\theta)}{\sin (\theta / 2) \sin (\theta)}=-2 \sin (n \theta) \cdot \frac{1-\cos (\theta)}{\sin (\theta)}
$$

and

$$
\alpha v R_{n ; 22}=2 \sin (\theta / 2) \frac{\alpha}{\sqrt{\Theta}} R_{n ; 22}
$$

Hence for all $n \in \mathbb{N}$,

$$
\left|\alpha v R_{n ; 21}\right| \leq 2
$$

and

$$
\left|\alpha v R_{n ; 22}\right| \leq 4 \sqrt{\epsilon^{2}+\Delta t}
$$

Statement 3. of the lemma follows from (33), (34) and (35).

Let us finally turn our attention to $\hat{f}_{0}=1$ and $\hat{g}_{0}(\xi, v)=\beta v$. We set

$$
P_{0}=1, \quad Q_{0}=1,
$$

and easily verify

$$
\hat{f}_{n}=P_{n}, \hat{g}_{n}=\beta v Q_{n}, \quad n \in \mathbb{N} \text { or } n=0,
$$

where for $n \in \mathbb{N}$ or $n=0$,

$$
\left(\begin{array}{c}
P_{n+1} \\
Q_{n+1}
\end{array}\right)=\left(\begin{array}{cc}
1 & -\Theta v^{2} \\
1 & 1-\Theta v^{2}
\end{array}\right)\left(\begin{array}{c}
P_{n} \\
Q_{n}
\end{array}\right),
$$


from which we obtain after some elementary manipulations for all $n \in \mathbb{N}$ (we recall $\Theta<2$ ),

$$
\begin{aligned}
& P_{n}=\operatorname{Re}\left(\left((1-a)-i \cdot \sqrt{2 a-a^{2}}\right)^{n} \cdot\left(1-\frac{i \cdot\left(2 a-a^{2}\right)}{\sqrt{2 a-a^{2}}}\right)\right), \\
& Q_{n}=\operatorname{Re}\left(\left((1-a)-i \cdot \sqrt{2 a-a^{2}}\right)^{n} \cdot\left(1+\frac{i \cdot(2-2 a)}{\sqrt{2 a-a^{2}}}\right)\right)
\end{aligned}
$$

where $a=\frac{\Theta v^{2}}{2}$ is as above. Then we have for all $n \in \mathbb{N}$,

$$
\left[(1, \alpha v) \cdot\left(\begin{array}{c}
\hat{f}_{n} \\
\hat{g}_{n}
\end{array}\right)\right]=\left[(1, \alpha v) \cdot\left(\begin{array}{c}
P_{n} \\
\beta v Q_{n}
\end{array}\right)\right]=\left[P_{n}-\Theta v^{2} Q_{n}\right]=\left[P_{n+1}\right] .
$$

Writing as above

$$
(1-a)+i \sqrt{2 a-a^{2}}=e^{i \theta}
$$

we have $\theta \in(0, \pi / 2), \cos (\theta)=(1-a), \sin (\theta)=\sqrt{2 a-a^{2}}$. Hence for all $n \in \mathbb{N}$,

$$
\begin{gathered}
P_{n}=\cos (n \theta)-\sin (n \theta) \frac{1-\cos (\theta)}{\sin (\theta)}, \\
Q_{n}=\cos (n \theta)+2 \sin (n \theta) \frac{1-\cos (\theta)}{\sin (\theta)},
\end{gathered}
$$

such that for all $n \in \mathbb{N}$,

$$
\left|\hat{f}_{n}\right|(\xi, v)=\left|P_{n}\right|(\xi, v) \leq 2,\left|\hat{g}_{n}\right|(\xi, v)=|\beta||v|\left|Q_{n}\right|(\xi, v) \leq 3|\beta| \leq 3|\xi|,
$$

and due to lemma 2 and due to (36) for all $n \in \mathbb{N}$,

$$
\begin{aligned}
& \int_{0}^{1}(1, \alpha s) \cdot\left(\begin{array}{c}
\hat{f}_{n}(\xi, s) \\
\hat{g_{n}}(\xi, s)
\end{array}\right) d s=\left[P_{n+1}\right](\xi) \\
= & \int_{0}^{1}\left(\cos ((n+1) \theta(s))-\sin ((n+1) \theta(s)) \frac{1-\cos (\theta(s))}{\sin (\theta(s))}\right) d s \in[-1,+1],
\end{aligned}
$$

where $\cos (\theta(s))=1-\frac{\Theta s^{2}}{2}, s \in(0,1)$.

\section{$5 \quad$ Proof of theorem 1}

For $n \in \mathbb{N}$ let $\hat{f}^{n}, \hat{g}^{n}$ be as in (13). In the sequel let $(\xi, v) \in \mathbb{R} \times(0,1)$ be fixed. We introduce for $n \in \mathbb{N}$ or $n=0$ the complex number

$$
\kappa_{n}:=B\left[\hat{f}^{n}+\alpha v \hat{g}^{n}\right](\xi) .
$$


Then it is easy to see that for all $n \in \mathbb{N}$,

$$
\left(\begin{array}{c}
\hat{f}^{n} \\
\hat{g}^{n}
\end{array}\right)=A^{n} T^{n}\left(\begin{array}{c}
\hat{f}^{0} \\
\hat{g}^{0}
\end{array}\right)+\sum_{j=0}^{n-1} \kappa_{j} A^{n-1-j} T^{n-1-j}\left(\begin{array}{c}
1 \\
\beta v
\end{array}\right) .
$$

We derive from (39) a recursion formula for $\left(\kappa_{n}\right)_{n \in \mathbb{N}}$,

$$
\begin{aligned}
\kappa_{0}= & B \cdot\left[\hat{f}^{0}+\alpha v \hat{g}^{0}\right](\xi) \\
\kappa_{n}= & B \cdot\left[(1, \alpha v) \cdot\left(\begin{array}{c}
\hat{f}^{n} \\
\hat{g}^{n}
\end{array}\right)\right] \\
= & A^{n} B \cdot\left[(1, \alpha v) \cdot\left(T^{n}\left(\begin{array}{c}
\hat{f}^{0} \\
\hat{g}^{0}
\end{array}\right)\right)\right] \\
& +\sum_{j=0}^{n-1} \kappa_{j} B A^{n-1-j}\left[(1, \alpha v) \cdot\left(T^{n-1-j}\left(\begin{array}{c}
1 \\
\beta v
\end{array}\right)\right)\right] \\
= & c_{n}+\left(\kappa_{0} \gamma_{n-1}+\ldots+\kappa_{n-1} \gamma_{0}\right)
\end{aligned}
$$

where for $n \in \mathbb{N}$,

$$
\begin{aligned}
& c_{n}:=A^{n} B\left[(1, \alpha v) \cdot\left(T^{n}\left(\begin{array}{c}
\hat{f}^{0} \\
\hat{g}^{0}
\end{array}\right)\right)\right](\xi), \\
& \gamma_{n}=B A^{n}\left[(1, \alpha v) \cdot\left(T^{n}\left(\begin{array}{c}
1 \\
\beta v
\end{array}\right)\right)\right](\xi) .
\end{aligned}
$$

By part 4 . of lemma 4 we have for all $n \in \mathbb{N}$

$$
\left|\gamma_{n}\right| \leq B A^{n}
$$

hence

$$
\sum_{n=0}^{\infty}\left|\gamma_{n}\right| \leq 1
$$

Furthermore, we have due to part 1 . and part 3 . of lemma 4 for all $n \in \mathbb{N}$,

$$
\begin{aligned}
\mid[(1, \alpha v) & \left.\cdot\left(T^{n}\left(\begin{array}{c}
\hat{f}^{0} \\
\hat{g}^{0}
\end{array}\right)\right)\right] \mid \\
\leq\left[2\left|\hat{f}^{0}\right|+\sqrt{2\left(\epsilon^{2}+\Delta t\right)}\left|\hat{g}^{0}\right|\right](\xi)+ & {\left[2\left|\hat{f}^{0}\right|+4 \sqrt{\epsilon^{2}+\Delta t}\left|\hat{g}^{0}\right|\right](\xi) } \\
& \leq 4\left[\left|\hat{f}^{0}\right|\right](\xi)+6 \sqrt{\epsilon^{2}+\Delta t}\left[\left|\hat{g}^{0}\right|\right](\xi)
\end{aligned}
$$


hence for all $n \in \mathbb{N}$,

$$
\left|c_{n}\right| \leq A^{n} B\left(4\left[\left|\hat{f}^{0}\right|\right](\xi)+6 \sqrt{\epsilon^{2}+\Delta t}\left[\left|\hat{g}^{0}\right|\right](\xi)\right),
$$

in particular $\left(c_{n}\right)_{n \in \mathbb{N}}$ is in $\ell^{1}(\mathbb{C})$.

We can therefore apply lemma 3 to deduce for all $n \in \mathbb{N}$ the estimate

$$
\begin{aligned}
&\left|\kappa_{n}\right| \leq\left|\kappa_{0}\right|+\left(\left|c_{0}\right|+\cdots+\left|c_{n-1}\right|\right) \\
& \leq\left|B\left[\hat{f}^{0}+\alpha v \hat{g}^{0}\right]\right|+\left(4\left[\left|\hat{f}^{0}\right|\right](\xi)+6 \sqrt{\epsilon^{2}+\Delta t}\left[\left|\hat{g}^{0}\right|\right](\xi)\right) \sum_{j=0}^{n-1} A^{n} B \\
& \leq\left[\left|\hat{f}^{0}\right|\right](\xi)+2 \sqrt{\epsilon^{2}+\Delta t}\left[\left|\hat{g}^{0}\right|\right](\xi)+4\left[\left|\hat{f}^{0}\right|\right](\xi)+6 \sqrt{\epsilon^{2}+\Delta t}\left[\left|\hat{g}^{0}\right|\right](\xi) \\
&=5\left[\left|\hat{f}^{0}\right|\right](\xi)+8 \sqrt{\epsilon^{2}+\Delta t}\left[\left|\hat{g}^{0}\right|\right](\xi)
\end{aligned}
$$

where we made use of the estimate

$$
|\alpha v| \leq 2 \sqrt{\epsilon^{2}+\Delta t}
$$

see (28).

Now we deduce from (39) via part 1 . of lemma 4 , via part 4 . of lemma 4 and via (42) for all $n \in \mathbb{N}$ the estimate

$$
\begin{aligned}
& \left|\hat{f}^{n}\right|(\xi, v) \\
& \quad \leq\left(2\left|\hat{f}^{0}\right|+\sqrt{2\left(\epsilon^{2}+\Delta t\right)}\left|\hat{g}^{0}\right|\right)(\xi, v)+10\left[\left|\hat{f}^{0}\right|\right](\xi)+16 \sqrt{\epsilon^{2}+\Delta t}\left[\left|\hat{g}^{0}\right|\right](\xi) .
\end{aligned}
$$

In a similiar way we deduce from (39) via part 2 . of lemma 4 , via part 4 . of lemma 4 and via (42) for all $n \in \mathbb{N}$ the estimate

$$
\begin{aligned}
& \left|\hat{g}^{n}\right|(\xi, v) \\
& \leq A\left(\frac{\sqrt{2}}{\sqrt{\epsilon^{2}+\Delta t}}\left|\hat{f}^{0}\right|+2\left|\hat{g}^{0}\right|\right)(\xi, v)+|\xi|\left(15\left[\left|\hat{f}^{0}\right|\right]+24 \sqrt{\epsilon^{2}+\Delta t}\left[\left|\hat{g}^{0}\right|\right]\right)(\xi) \\
& \leq\left(\frac{\sqrt{2} \epsilon^{2}}{\left(\epsilon^{2}+\Delta t\right)^{3 / 2}}\left|\hat{f}^{0}\right|+2\left|\hat{g}^{0}\right|\right)(\xi, v)+|\xi|\left(15\left[\left|\hat{f}^{0}\right|\right]+24 \sqrt{\epsilon^{2}+\Delta t}\left[\left|\hat{g}^{0}\right|\right]\right)(\xi) .
\end{aligned}
$$


We deduce

$$
\begin{aligned}
& \left\|\mathfrak{f}^{n}\right\|_{2}=\left\|\hat{\mathfrak{f}}^{n}\right\|_{2} \leq\left\|\hat{f}^{n}\right\|_{\mathcal{L}^{2}(\mathbb{R} \times(0,1))}+\left\|\hat{g}^{n}\right\|_{\mathcal{L}^{2}(\mathbb{R} \times(0,1))} \\
& \leq 2\left\|\hat{f}^{0}\right\|_{\mathcal{L}^{2}(\mathbb{R} \times(0,1))}+\sqrt{2\left(\epsilon^{2}+\Delta t\right)}\left\|\hat{g}^{0}\right\|_{\mathcal{L}^{2}(\mathbb{R} \times(0,1))} \\
& +10\left\|\hat{f}^{0}\right\|_{\mathcal{L}^{2}(\mathbb{R} \times(0,1))}+16 \sqrt{\epsilon^{2}+\Delta t}\left\|\hat{g}^{0}\right\|_{\mathcal{L}^{2}(\mathbb{R} \times(0,1))} \\
& +\frac{\sqrt{2} \epsilon^{2}}{\left(\epsilon^{2}+\Delta t\right)^{3 / 2}}\left\|\hat{f}^{0}\right\|_{\mathcal{L}^{2}(\mathbb{R} \times(0,1))}+2\left\|\hat{g}^{0}\right\|_{\mathcal{L}^{2}(\mathbb{R} \times(0,1))} \\
& +15 \sqrt{\int_{\mathbb{R} \times(0,1)}|\xi|^{2}\left|\hat{f}^{0}\right|^{2}(\xi, v) d(\xi, v)}+24 \sqrt{\epsilon^{2}+\Delta t} \sqrt{\int_{\mathbb{R} \times(0,1)}|\xi|^{2}\left|\hat{g}^{0}\right|^{2}(\xi, v) d(\xi, v)} \\
& \leq\left(15+\frac{\sqrt{2} \epsilon^{2}}{\left(\epsilon^{2}+\Delta t\right)^{3 / 2}}\right)\left(\left\|\hat{f}^{0}\right\|_{\mathcal{L}^{2}(\mathbb{R} \times(0,1))}+\sqrt{\int_{\mathbb{R} \times(0,1)}|\xi|^{2}\left|\hat{f}^{0}\right|^{2}(\xi, v) d(\xi, v)}\right) \\
& +\left(2+42 \sqrt{\epsilon^{2}+\Delta t}\right)\left(\left\|\hat{g}^{0}\right\|_{\mathcal{L}^{2}(\mathbb{R} \times(0,1))}+\sqrt{\int_{\mathbb{R} \times(0,1)}|\xi|^{2}\left|\hat{g}^{0}\right|^{2}(\xi, v) d(\xi, v)}\right) \\
& \leq \sqrt{2}\left(42 \sqrt{\epsilon^{2}+\Delta t}+15+\frac{\sqrt{2} \epsilon^{2}}{\left(\epsilon^{2}+\Delta t\right)^{3 / 2}}\right) \\
& \times\left(\sqrt{\int_{\mathbb{R} \times(0,1)}\left(1+|\xi|^{2}\right)\left|\hat{f}^{0}\right|^{2}(\xi, v) d(\xi, v)}+\sqrt{\int_{\mathbb{R} \times(0,1)}\left(1+|\xi|^{2}\right)\left|\hat{g}^{0}\right|^{2}(\xi, v) d(\xi, v)}\right) \\
& \leq 2\left(42 \sqrt{\epsilon^{2}+\Delta t}+15+\frac{\sqrt{2} \epsilon^{2}}{\left(\epsilon^{2}+\Delta t\right)^{3 / 2}}\right) \\
& \times \sqrt{\int_{\mathbb{R} \times(0,1)}\left(1+|\xi|^{2}\right)\left(\left|\hat{f}^{0}\right|^{2}+\left|\hat{g}^{0}\right|^{2}\right)(\xi, v) d(\xi, v)} \\
& =2\left(42 \sqrt{\epsilon^{2}+\Delta t}+15+\frac{\sqrt{2} \epsilon^{2}}{\left(\epsilon^{2}+\Delta t\right)^{3 / 2}}\right)\left\|\mathfrak{f}^{0}\right\|_{1 ; 1} .
\end{aligned}
$$


On the other hand, we have for each $v \in(0,1)$,

$$
\begin{aligned}
& \sqrt{\int_{\mathbb{R}}\left(\left|f^{n}\right|^{2}+\left|g^{n}\right|^{2}\right)(\xi, v) d \xi}=\sqrt{\int_{\mathbb{R}}\left(\left|\hat{f}^{n}\right|^{2}+\left|\hat{g}^{n}\right|^{2}\right)(\xi, v) d \xi} \\
& \leq \sqrt{\int_{\mathbb{R}}\left|\hat{f}^{n}\right|^{2}(\xi, v) d \xi}+\sqrt{\int_{\mathbb{R}}\left|\hat{g}^{n}\right|^{2}(\xi, v) d \xi}=\left\|\hat{f}^{n}(., v)\right\|_{\mathcal{L}^{2}(\mathbb{R})}+\left\|\hat{g}^{n}(., v)\right\|_{\mathcal{L}^{2}(\mathbb{R})} \\
& \leq 2\left\|\hat{f}^{0}(., v)\right\|_{\mathcal{L}^{2}(\mathbb{R})}+\sqrt{2\left(\epsilon^{2}+\Delta t\right)}\left\|\hat{g}^{0}(., v)\right\|_{\mathcal{L}^{2}(\mathbb{R})}+10\left\|\hat{f}^{0}\right\|_{\mathcal{L}^{2}(\mathbb{R} \times(0,1))} \\
& +16 \sqrt{\epsilon^{2}+\Delta t}\left\|\hat{g}^{0}\right\|_{\mathcal{L}^{2}(\mathbb{R} \times(0,1))}+\frac{\sqrt{2} \epsilon^{2}}{\left(\epsilon^{2}+\Delta t\right)^{3 / 2}}\left\|\hat{f}^{0}(., v)\right\|_{\mathcal{L}^{2}(\mathbb{R})}+2\left\|\hat{g}^{0}(., v)\right\|_{\mathcal{L}^{2}(\mathbb{R})} \\
& +15 \sqrt{\int_{\mathbb{R} \times(0,1)}|\xi|^{2}\left|\hat{f}^{0}\right|^{2}(\xi, v) d(\xi, v)}+24 \sqrt{\epsilon^{2}+\Delta t} \sqrt{\int_{\mathbb{R} \times(0,1)}|\xi|^{2}\left|\hat{g}^{0}\right|^{2}(\xi, v) d(\xi, v)} \\
& \leq\left(2+2 \sqrt{\epsilon^{2}+\Delta t}+\frac{\sqrt{2} \epsilon^{2}}{\left(\epsilon^{2}+\Delta t\right)^{3 / 2}}\right)\left(\left\|\hat{f}^{0}(., v)\right\|_{\mathcal{L}^{2}(\mathbb{R})}+\left\|\hat{g}^{0}(., v)\right\|_{\mathcal{L}^{2}(\mathbb{R})}\right) \\
& +15\left(\left\|\hat{f}^{0}\right\|_{\mathcal{L}^{2}(\mathbb{R} \times(0,1))}+\sqrt{\int_{\mathbb{R} \times(0,1)}|\xi|^{2}\left|\hat{f}^{0}\right|^{2}(\xi, v) d(\xi, v)}\right) \\
& +24 \sqrt{\epsilon^{2}+\Delta t}\left(\left\|\hat{g}^{0}\right\|_{\mathcal{L}^{2}(\mathbb{R} \times(0,1))}+\sqrt{\int_{\mathbb{R} \times(0,1)}|\xi|^{2}\left|\hat{g}^{0}\right|^{2}(\xi, v) d(\xi, v)}\right) \\
& \leq \sqrt{2}\left(2+2 \sqrt{\epsilon^{2}+\Delta t}+\frac{\sqrt{2} \epsilon^{2}}{\left(\epsilon^{2}+\Delta t\right)^{3 / 2}}\right) \sqrt{\int_{\mathbb{R}}\left(\left|\hat{f}^{n}\right|^{2}+\left|\hat{g}^{n}\right|^{2}\right)(\xi, v) d \xi} \\
& +2\left(15+24 \sqrt{\epsilon^{2}+\Delta t}\right) \sqrt{\int_{\mathbb{R} \times(0,1)}\left(1+|\xi|^{2}\right)\left(\left|\hat{f}^{0}\right|^{2}+\left|\hat{g}^{0}\right|^{2}\right)(\xi, v) d(\xi, v)} \\
& =\sqrt{2}\left(2+2 \sqrt{\epsilon^{2}+\Delta t}+\frac{\sqrt{2} \epsilon^{2}}{\left(\epsilon^{2}+\Delta t\right)^{3 / 2}}\right) \sqrt{\int_{\mathbb{R}}\left(\left|f^{n}\right|^{2}+\left|g^{n}\right|^{2}\right)(\xi, v) d \xi} \\
& +2\left(15+24 \sqrt{\epsilon^{2}+\Delta t}\right)\left\|\mathfrak{f}^{0}\right\|_{1 ; 1} \\
& \leq \sqrt{2}\left(2+2 \sqrt{\epsilon^{2}+\Delta t}+\frac{\sqrt{2} \epsilon^{2}}{\left(\epsilon^{2}+\Delta t\right)^{3 / 2}}\right)\left\|\mathfrak{f}^{0}\right\|_{0 ; \infty}++2\left(15+24 \sqrt{\epsilon^{2}+\Delta t}\right)\left\|\mathfrak{f}^{0}\right\|_{1 ; 1} \text {. }
\end{aligned}
$$

Taking the supremum with respect to $v \in(0,1)$ on the left hand side of this inequality establishes b) of theorem 1 .

\section{Conclusions}

We have proved uniform stability of the iterative scheme under two restrictions: 
- Uniform boundedness of the iterative scheme could be proven for underresolved numerical computations $\epsilon \leq \epsilon_{0} \Delta t$ or bounded mean free path $\epsilon_{1} \leq$ $\epsilon \leq M$.

- The necessary CFL restriction is in the diffusive limit a parabolic CFL condition as was to be expected. However, for finite values of $\epsilon$ the parabolic restriction can be relaxed. One obtains a CFL condition adapted to the hyperbolic part of the original kinetic equation.

\section{References}

[1] R.E. Caflisch, S. Jin, and G. Russo. Uniformly accurate schemes for hyperbolic systems with relaxation. SIAM J. Num. Anal., 34:246-281, 1997.

[2] F. Golse, S. Jin, and D. Levermore. The convergence of numerical transfer schemes in diffusive regimes II: The cell edge even parity scheme. Preprint.

[3] F. Golse, S. Jin, and D. Levermore. The convergence of numerical transfer schemes in diffusive regimes I: Discrete ordinate method. SINUM, 36:13331369, 1999.

[4] S. Jin. Efficient asymptotic-preserving schemes for some multiscale kinetic equations. Preprint, 1998.

[5] S. Jin, L. Pareschi, and G. Toscani. Diffusive relaxation schemes for discretevelocity kinetic equations. SIAM J. Num. Anal., 35:2405-2439, 1998.

[6] S. Jin, L. Pareschi, and G. Toscani. Uniformly accurate diffusive relaxation schemes for multiscale transport equations. preprint, 1999.

[7] A. Klar. An asymptotic-induced scheme for nonstationary transport equations in the diffusive limit. SIAM J. Num. Anal., 35:1073-1094, 1998.

[8] A. Klar. Relaxation schemes for a Lattice Boltzmann type discrete velocity model and numerical Navier Stokes limit. J. Comp. Phys., 148:1-17, 1999.

[9] A. Klar and C. Schmeiser. Numerical passage from radiative heat transfer to nonlinear diffusion models. preprint, 1998.

[10] E.W. Larsen, J.E. Morel, and W.F. Miller. Asymptotic solution of numerical transport problems in optically thick, diffusive regimes. J. Comp. Phys., 69:283-324, 1987.

[11] C Schmeiser and A. Zwirchmayr. Convergence of moment methods for the semiconductor Boltzmann equation. SIAM J. Num. Anal., to appear. 